ISSN: 2215-2644

revedu@gmail.com

Universidad de Costa Rica

Costa Rica

\title{
Desarrollo del pensamiento computacional y las competencias matemáticas en análisis y solución de problemas: una experiencia de aprendizaje con Scratch en la plataforma Moodle
}

Cabra Páez, Mercy Liceth; Ramírez Gamboa, Sergio Albeiro

Desarrollo del pensamiento computacional y las competencias matemáticas en análisis y solución de problemas: una experiencia de aprendizaje con Scratch en la plataforma Moodle

Revista Educación, vol. 46, núm. 1, 2022

Universidad de Costa Rica, Costa Rica

Disponible en: https://www.redalyc.org/articulo.oa?id=44068165016

DOI: https://doi.org/10.15517/revedu.v46i1.44970

\section{(c) $(1) \Theta \Theta$}

Esta obra está bajo una Licencia Creative Commons Atribución-NoComercial-SinDerivar 3.0 Internacional. 


\title{
Desarrollo del pensamiento computacional y las competencias matemáticas en análisis y solución de problemas: una experiencia de aprendizaje con Scratch en la plataforma Moodle
}

\author{
Developing Computational Thinking and Mathematical Skills for Analysis and Problem Solving: Learning on \\ Scratch Moodle Platforms
}

Mercy Liceth Cabra Páez

Institución Educativa Departamental Santa María, Colombia

iedsantamaria@gmail.com

iD https://orcid.org/0000-0002-7199-3893

Sergio Albeiro Ramirez Gamboa

Universidad de Santander, Bucaramanga, Colombia

sergio.ramirez@cvudes.edu.co

(iD https://orcid.org/0000-0002-0396-3609
DOI: https://doi.org/10.15517/revedu.v46i1.44970

Redalyc: https://www.redalyc.org/articulo.oa?

$\mathrm{id}=44068165016$

\section{Resumen:}

La propuesta investigativa surgió para demostrar el fortalecimiento de las competencias matemáticas en análisis y solución de problemas cotidianos que involucran las cuatro operaciones básicas y el desarrollo del pensamiento computacional, aplicados a la vida cotidiana, contextualizados al entorno de 20 estudiantes del grado 305 de la Institución Educativa Departamental (IED) Santa María del municipio de Ubaté Cundinamarca, de forma clara, eficiente y sencilla, por medio del curso denominado: Pensamiento computacional y resolución de problemas matemáticos con Scratch en la plataforma Moodle. Se diseñaron los instrumentos para la recolección de la información (encuesta de caracterización socio tecnológica, prueba diagnóstica, prueba final, encuesta de percepción del curso) y se aplicó la propuesta. Se tomó en cuenta la metodología basada en el enfoque mixto, con un diseño exploratorio secuencial por medio del proceso de recolección, análisis y vinculación de datos cualitativos y cuantitativos, en coherencia con el modelo STEAM. Se realizó el análisis comparativo de las pruebas diagnóstica y final, en las que se identificaron las dificultades del estudiantado para resolver problemas con las operaciones básicas; posteriormente, se evidenció un avance y mejoramiento reflejado en puntajes y promedios considerables, con un progreso significativo, principalmente en la evaluación final, pues se presentó un conjunto de actividades en la que se desarrollaron ejercicios de algoritmos y procedimientos por medio de Scratch. Los resultados demostraron que la aplicación de la estrategia pedagógica Scratch en la plataforma Moodle mejoró significativamente el desarrollo de las competencias matemáticas y el pensamiento computacional del estudiantado de tercero de primaria.

Palabras Clave: Pensamiento computacional, Competencias Matemáticas, STEAM, Operaciones básicas, Scratch, Moodle.

\section{AbStract:}

This study demonstrates how strengthening mathematical skills enables analysis and resolution of real, everyday-life problems by using the four basic math operations to develop computational thinking. The study participants included 20 third grade students from the Santa María Secondary School (Colegio Santa María), an IED (Departmental Educational Institution) located in Ubate, Cundinamarca, all enrolled in a Scratch and Moodle-based course called Computational Thinking and Mathematical Problem Solving. Data-collection tools included a socio-technological characterization survey, a diagnostic and final exam as well as a course perception survey. The methodology consisted of a combined approach with a sequential exploratory design to collect, analyze and link qualitative and quantitative data consistent with the STEAM model. A comparative analysis of the diagnostic and final exams identified student difficulties in resolving problems using basic operations. Progress in the form of improved scores was detected after the final assessment. This was noted after student presentations showing activities and algorithms developed using Scratchbased programs. Based on the results, the use of a pedagogical Scratch strategy on the Moodle platform significantly improved mathematical skills and computational thinking among third grade students. 
KEYWORDs: Computational Thinking, Mathematical Skills, STEAM, Basic Operations, Scratch, Moodle.

\section{INTRODUCCIÓN}

La propuesta investigativa, basada en el análisis y resolución de problemas que integran las cuatro operaciones básicas (suma, resta, multiplicación y división) y el desarrollo del pensamiento computacional, consistió en la implementación de la estrategia pedagógica y tecnológica de Scratch en el ambiente virtual de aprendizaje Moodle, el cual contribuyó con el fortalecimiento de las competencias matemáticas de estudiantes del grado tercero de la Institución Educativa Departamental Santa María del municipio de Ubaté Cundinamarca.

Se basó en una investigación enfocada hacia el aprovechamiento de la curiosidad y la atracción por el desarrollo de acciones pedagógicas basadas en la innovación por parte del alumnado, y que funcionara como herramienta para favorecer los resultados en el proceso de enseñanza aprendizaje apoyados en el modelo STEAM (Ciencia, Tecnología, Ingeniería, Arte y Matemáticas).

En consideración con lo anterior, se realizó una revisión bibliográfica para identificar el estado del arte de la presente investigación en coherencia con otras ya desarrolladas, tanto a nivel internacional como a nivel nacional, en el que se encontraron aportes significativos.

A nivel internacional, Basogain-Olabe et al. (2015), España, Madrid, en su artículo presentan el concepto de pensamiento computacional y la forma de integrarlo en el aula a través del diseño e implementación de proyectos de programación en Scratch, lenguaje de programación y herramienta que favorece el desarrollo de competencias y habilidades para analizar y resolver problemas.

Cabe resaltar que, al mismo tiempo, González (2019), España, Salamanca, en su artículo realiza un análisis a las principales iniciativas relacionadas con el pensamiento computacional en las escuelas, lo cual quiere decir que "el pensamiento computacional, estrechamente relacionado con la programación, requiere pensar y resolver problemas con diferentes niveles de abstracción” (González, 2019, p. 17) para fortalecer las competencias matemáticas básicas del estudiantado de grado tercero.

Dentro de este contexto, Álvarez (2017), España Castilla, en su trabajo de investigación, pretende evaluar la factibilidad del desarrollo del pensamiento computacional (PC) a partir de la innovación y la integración de las TIC; el estudiantado tendrá la oportunidad de interactuar de manera directa con el lenguaje de programación, ya sea para recrear o desarrollar problemas matemáticos.

Por consiguiente, Mendoza (2017), Perú, Chincha, en su trabajo de investigación, propone demostrar que, la aplicación del software de programación, Scratch permite desarrollar el pensamiento lógico matemático y, por tal motivo, se considera de gran importancia, ya que aporta de manera significativa en el proceso de análisis y en el de comprensión de la solución de problemas matemáticos.

Más aún, Granados (2019), Perú, Lima, en su trabajo de investigación, propone demostrar la influencia del programa Scratch en la resolución de problemas en el área de matemática y responde a la problemática institucional con la viabilidad para implementar dicha estrategia en el proceso de enseñanza que fortalezca las competencias matemáticas.

Por otro lado, Quintero (2019), Perú, Tacna, en su tesis de maestría en Tecnología Educativa, propone determinar la influencia Scratch en el pensamiento computacional del estudiantado, con lo que permite concluir que el programa influye significativamente sobre el pensamiento computacional, sobre todo en lo que hace referencia al fortalecimiento del pensamiento numérico.

A la vez, a nivel nacional, Rios (2015), Colombia, Medellín, en su tesis de maestría denominada Scratch + ABP, como estrategia para el desarrollo del pensamiento computacional, plantea una estrategia metodológica basada en el uso del programa Scratch + A.B.P (aprendizaje basado en problemas) como alternativa adecuada para el desarrollo de competencias de pensamiento computacional y competencias matemáticas. 
Por su parte, Aristizabal (2016), Colombia, Quindío, en su artículo hace un análisis exhaustivo sobre la importancia del desarrollo de las habilidades y relaciones para que el estudiantado de grado quinto de primaria se familiarice y refuerce las operaciones básicas (adición, sustracción, multiplicación y división) por medio del juego y la interacción con herramientas tecnológicas.

También, Pérez (2017) aporta de manera científica que:

A la didáctica de las tecnologías de la información y la comunicación, desde el cual se evidencian las potencialidades y privilegios pedagógicos que ofrecen las ciencias de la computación para el desarrollo de competencias relacionadas con la resolución creativa de problemas. (p. 1).

Asimismo, Lagos (2017), Colombia, Bogotá, en su proyecto de investigación, hace una intervención de clases basada en situaciones de estructura aditiva para contribuir en la comprensión del concepto de número y evidenciar las actitudes del estudiantado en la resolución de situaciones aditivas.

Por ello, el desarrollo de las competencias matemáticas por medio del pensamiento computacional contribuye a desarrollar la creatividad y habilidades para la solución de problemas y permite que el alumnado encuentre motivación y se sienta autosuficiente al trabajar en entornos virtuales de aprendizaje acordes al nivel educativo, como el lenguaje de programación Scratch, planteado como estrategia para fortalecer las competencias matemáticas en la presente propuesta.

Es así como, Suarez (2019), Colombia, Cali, en su trabajo de investigación dirigido a estudiantes del nivel de primaria, considera que es de suma importancia investigar los recursos tecnológicos más apropiados para implementarlos en el aula, de manera que contribuyan significativamente con el fortalecimiento de las dificultades de comprensión en el proceso de resolución de problemas matemáticos.

De igual forma, Acuña et al. (2018), Colombia, Magdalena, en su tesis de maestría en educación, pretende ofrecer una estrategia para mejorar el aprendizaje de las operaciones básicas de matemáticas al mediar el conocimiento con el uso de las tecnologías de información y comunicación (TIC); por esto, se debe aprovechar la variada posibilidad de conocimiento por enseñar, aprender y contribuir con la calidad educativa.

Finalmente, Vivas (2019), Colombia, Tunja, en su artículo de investigación dirigido a estudiantes de escuela nueva, indica:

La necesidad de fortalecer procesos multiplicativos y favorecer el pensamiento numérico en la formación integral del educando para reforzar el aprendizaje en la resolución de operaciones básicas y contribuir a desarrollar habilidades que respondan con eficiencia a solucionar problemas matemáticos. (p.1).

Lo anterior permite evidenciar que las personas autoras reconocen que hoy en día existe variedad de herramientas tecnológicas, las cuales contribuyen de manera significativa al integrarlas en el proceso de enseñanza aprendizaje y en el currículo escolar por medio del uso de instrumentos y lenguajes de programación, esto brinda competencias esenciales para mejorar la motivación, la autonomía y fomentar la creatividad en el aula de clase.

En consecuencia, se determinó una muestra de 200 estudiantes niñas entre los 7 y 10 años de edad, del grado tercero de primaria. Se planteó el proceso investigativo en cuatro fases, las cuales hacen referencia a: Fase 1. Diagnóstico y exploración; Fase 2. Diseño de la estrategia pedagógica y del AVA; Fase 3. Implementación; Fase 4. Evaluación de los resultados obtenidos.

En lo que corresponde a la primera fase de la investigación, se planteó el diseño y aplicación de entrevistas, una encuesta de caracterización de la población objeto de estudio y una prueba diagnóstica para identificar las dificultades específicas del grupo de estudiantes en las competencias matemáticas, esto de acuerdo con el aporte de los contenidos en los estándares básicos de competencia, los derechos básicos de aprendizaje y las matrices de referencia del área de matemáticas del nivel primario, más específicamente del grado tercero, según los planteamientos del Ministerio de Educación Nacional (MEN, 2016), lo cual permitió la consolidación del diagnóstico, que sirvió como línea para implementar la propuesta. 
En la segunda fase se diseñó la estrategia pedagógica y el AVA, en coherencia con la articulación de las necesidades de las estudiantes con los componentes pedagógicos, esto por medio de la creación de actividades didácticas y ejercicios interactivos de problemas matemáticos y con el uso de las herramientas TIC.

En la tercera fase se implementa la estrategia pedagógica en el AVA, de forma virtual, por medio de la interacción directa de las estudiantes en el desarrollo de las diferentes actividades creadas por la persona docente, para generar un impacto significativo en el proceso de formación integral y en consideración de las necesidades e intereses particulares, así como el desarrollo del pensamiento computacional.

Finalmente, en la cuarta y última fase, se determinó la efectividad de la estrategia implementada mediante la valoración de actividades realizadas por las estudiantes durante la implementación de la estrategia e interacción con el AVA, y evaluación de las competencias matemáticas de las estudiantes en análisis y resolución de problemas que involucran las operaciones básicas.

La investigación aportó de manera significativa al enriquecimiento del modelo pedagógico constructivista de la Institución Educativa Santa María; se estableció como una estrategia a incluir dentro del plan de mejoramiento y seguimiento académico de la gestión en el aula, así como en el diseño e integración curricular para el área de matemáticas y la propuesta de investigación.

De igual forma, brindó una alternativa metodológica, no solo para el estudiantado, sino también para el profesorado a la hora de impartir los conocimientos, basada en la enseñanza problemática; también se ofrecieron las consideraciones necesarias, según el criterio de las personas autoras, a la hora de resolver determinado problema, pues las estudiantes estudiaron conceptos básicos matemáticos, definiciones y varios procedimientos utilizados para resolver problemas.

Vale la pena reconocer que en esta investigación a Halmos (1980), quien establece: "la resolución de problemas ha sido reconocida como un componente importante en el estudio del conocimiento matemático" (p.1).

Por su parte, Barbarán (2014) sugirió que "resolver problemas es el corazón de las matemáticas y la principal razón de existir del matemático es resolver problemas, y por lo tanto en lo que realmente consisten las matemáticas es en problemas y soluciones" (p.3).

Con base en esto, se analizó que no fue una tarea fácil, pues para el desarrollo de cada estrategia surgen algunas dificultades que en el camino se deben fortalecer. Por lo tanto, se debe tener cuidado al momento de elegir los problemas, ya que se puede generar tanta motivación como indiferencia en el alumnado; asimismo, mirar el contexto más apropiado y cercano al estudiantado permite lograr resultados satisfactorios y hacer que los aprendizajes sean más significativos. Por tal motivo, se puede decir que la propuesta investigativa fue novedosa, por cuanto permitió evidenciar los problemas del desarrollo del pensamiento numérico y cómo buscar la solución a estos mismos.

También, Guzmán y López (2029) resaltan "la importancia de la programación y el desarrollo de las habilidades tecnológicas, como complemento de las TIC para el desarrollo del pensamiento computacional aplicado en edades tempranas del desarrollo cognoscitivo" (p.36), ya que, según Álvarez (2017), "cada vez son más las herramientas que trabajan la programación informática, aunque en la actualidad la herramienta más destacable es Scratch" (p.2). Por esto, se facilitó la implementación de dicha estrategia interactiva de aprendizaje, para que las estudiantes desarrollen distintas actividades creadas por la docente y, de igual forma aprendieran a crear otras similares, lo cual generó el desarrollo de la creatividad del estudiantado y personal docente participante para afianzar el proceso de enseñanza aprendizaje.

Asimismo, Guzmán y López (2009) afirman que:

El intervalo de tiempo en el que las estudiantes apropiaron los conocimientos ofrecidos desde el contexto familiar y escolar, brindó espacios significativos con herramientas que potencializan el nivel de aprendizaje y generan nuevas experiencias a partir de la interacción con la realidad inmediata. De esta manera, lograron madurar el pensamiento crítico y reflexivo al asumir o proponer solución a las situaciones que se presenten. (p.34). 
Lo anterior de acuerdo con los objetivos establecidos y al realizar la propuesta pedagógica según a las necesidades identificadas y con el uso herramientas TIC; también con el análisis posterior de la efectividad de la utilización de la metodología planteada. De igual forma, se comprobó que la estrategia pedagógica fue pertinente, por lo que se puede tener en cuenta en investigaciones futuras y a partir de los resultados obtenidos, y que al mismo tiempo se replanteen alternativas de aprendizaje que generen procesos de articulación del conocimiento de forma presencial y virtual con un mismo nivel de importancia.

\section{Procedimiento metodológico}

La propuesta de investigación se planteó con el enfoque mixto, pues, según Hernández-Sampieri y Mendoza (2008),

Representa un conjunto de procesos sistemáticos, empíricos y críticos de investigación e implican la recolección y el análisis de datos cuantitativos y cualitativos, así como su integración y discusión conjunta, para realizar inferencias producto de toda la información recaudada (meta inferencias) y lograr un mayor entendimiento del fenómeno bajo estudio (p.3)

Por lo tanto, implicó un proceso de recolección, análisis y vinculación de datos cualitativos y cuantitativos para responder al planteamiento del problema formulado, relacionado con el fortalecimiento de las competencias matemáticas en análisis y resolución de problemas que integran las operaciones básicas, y la medición del rendimiento académico del estudiantado. También, se tuvo presente que la investigación hoy en día "necesita de un trabajo multidisciplinario, lo cual contribuye a que se realice en equipos integrados por personas con intereses y aproximaciones metodológicas diversas, que refuerza la necesidad de usar diseños multimodales" (Creswell, 2009. p.2). Por tal motivo, se examinaron las características, posibilidades y ventajas orientadas al proceso de analizar, comprender, explorar y evaluar el modo particular que tiene la Institución Educativa Departamental Santa María, Sede María Auxiliadora, del municipio de Ubaté, para adoptar la implementación de las TIC en el desarrollo de las clases, principalmente del área de matemáticas.

Para lograr lo anterior, se hizo un acercamiento de observación directa al rendimiento académico del estudiantado participante del grado tercero en dicha área del conocimiento, además de elaborar diferentes instrumentos de recolección de información de tipo cuantitativo y cualitativo, que permitieron identificar situaciones particulares y fortalecer las prácticas pedagógicas mediadas por TIC. Lo anterior respondió a las necesidades de la investigación, pues reconoció la apropiación de las TIC en la IE y presentó características únicas y particulares, como estrategias investigativas y prácticas, en coherencia con los planteamientos de Creswell (2009), quien comenta que este diseño "es apropiado cuando buscamos probar elementos de una teoría emergente producto de la fase cualitativa y pretendemos generalizarla a diferentes muestras" (p.6). En este sentido, Hernández-Sampieri y Mendoza (2008) afirman que dicho enfoque "es utilizado cuando el investigador necesita desarrollar un instrumento estandarizado porque las herramientas existentes son inadecuadas o no se puede disponer de ellas, y en este caso fue útil usar un diseño exploratorio secuencial” (p.551), siempre en consideración de cada una de las etapas de recolección de datos, análisis y uso de resultados y administración de los instrumentos para validar el proceso investigativo.

$\mathrm{Al}$ respecto se realizó una prueba interna diagnóstica, implementada específicamente al estudiantado de grado tercero, en la que se identificaron dificultades en el desarrollo de habilidades de análisis, comprensión y resolución de problemas matemáticos durante el proceso de aprendizaje; además, se desarrollaron cuatro preguntas y actividades específicamente relacionadas con el análisis y resolución de problemas de suma, resta, multiplicación y división, en la que se evidencia claramente el porcentaje de estudiantes que presenta dicha dificultad.

Asimismo, se diseñaron varios instrumentos para la recolección de información: la encuesta de caracterización socio tecnológica, definida como "una técnica que pretende obtener información que suministra un grupo o muestra de sujetos acerca de sí mismos o en relación con un tema en particular” (Arias, 
2012, p. 67), debido a que se identifican los distintos factores socioeconómicos y socio tecnológicos sobre las condiciones particulares de cada estudiante y el contexto familiar. Dicho instrumento, conformado por 20 preguntas, se aplicó al estudiantado objeto de estudio antes de la implementación de la estrategia, específicamente en las casas de cada niña con la orientación virtual de la docente investigadora, esto por la emergencia sanitaria Covid19 vivida a nivel mundial.

Luego, se aplicó la prueba diagnóstica para identificar las habilidades lógicas, matemáticas, tecnológicas y de resolución de problemas, y así evidenciar el antes y después de la aplicación de dichos instrumentos, que permitieron identificar los procesos reales y avances significativos para realizar los respectivos análisis de información, diseñada con base a las competencias del tema o asignatura propuesta y sobre pensamiento computacional; esta se conformaba de 15 preguntas divididas en dos partes. La primera para hacer un diagnóstico de las competencias matemáticas en análisis y resolución de problemas que integran las cuatro operaciones básicas; la segunda, sobre preguntas de pensamiento computacional como instrumento a utilizar para el fortalecimiento de dichas competencias.

Enseguida, se implementó la prueba final, para ver el progreso del estudiantado; se aplicó la misma cantidad de preguntas que en la prueba diagnóstica, pero con mayor complejidad, ya que incluyen la estrategia TIC y el ambiente virtual de aprendizaje para evaluar el nivel de comprensión de los aprendizajes adquiridos por el estudiantado.

También se aplicó la encuesta de satisfacción, para evaluar el nivel de comprensión y fortalecimiento de las competencias matemáticas en análisis y resolución de problemas por medio de la interacción directa de las estudiantes del grado tercero con Scratch y Moodle; fotografías y documentos para realizar un análisis cualitativo y cuantitativo, para la recolección de información sobre las competencias matemáticas y tecnológicas en análisis y resolución de problemas en estudiantes del grado tercero de la Institución Educativa Departamental (IED) Santa María de Ubaté, Cundinamarca; dichos instrumentos se aplicaron en el segundo semestre del año 2020; se tomó en cuenta el aporte de los contenidos en los Estándares Básicos de Competencia, los Derechos básicos de aprendizaje y las matrices de referencia del área de matemáticas del nivel primario, más específicamente del grado tercero, según los planteamientos del MEN (2016). De igual forma, se utilizaron las guías digitales y didácticas de matemáticas, con contenidos de aprendizaje basados en el análisis y solución de problemas de integración curricular STEAM, que involucran las cuatro operaciones matemáticas básicas.

Según la implementación de cada uno de los instrumentos para la recolección de la información pertinente —aplicados a la totalidad de la población objeto de estudio, correspondiente a 200 estudiantes del grado tercero-, se realizó el análisis descriptivo y estadístico, en el cual se evidencian los resultados a través de tablas, gráficas y porcentajes específicos. Con la ayuda de Excel se realiza la tabulación y gráficos de los instrumentos mediante técnicas cuantitativas. Luego se hace el análisis cualitativo de forma exploratoria y descriptiva con base en las gráficas. Cabe resaltar que los cuatro instrumentos para la recolección de la información se diseñaron en los formularios de Google, los cuales favorecieron de manera significativa al proceso de análisis y tabulación cuantitativa y cualitativa de las respuestas.

Finalmente, para validar y complementar la información respectiva —en cuanto a los resultados arrojados a través de los distintos análisis estadísticos sobre el desempeño individual del alumnado en el desarrollo de las competencias matemáticas y el pensamiento computacional- se diseñan las cinco rúbricas de evaluación correspondientes, según Espinosa (2014) "las más adecuadas son las que admiten valoración central, partiendo de un mínimo de tres niveles y un máximo de cinco, con una graduación lógica para que el proceso de evaluación sea eficiente" (p.4). Por tal motivo, para la investigación se planteó cada rúbrica con 4 criterios para tener en cuenta en el proceso evaluativo para el desarrollo de cada actividad, en la cual se estableció el rango de valoración de 10 a 50; con base en ellas se retroalimentó el desempeño individual del estudiantado. 


\section{ANÁLISIS Y DISCUSIÓN DE RESULTADOS}

En el desarrollo de este trabajo se identificaron, por medio de la prueba interna, las necesidades educativas de la población, según el nivel de desarrollo de competencias matemáticas basadas en la resolución de problemas que integran las cuatro operaciones básicas, como se muestra a continuación.

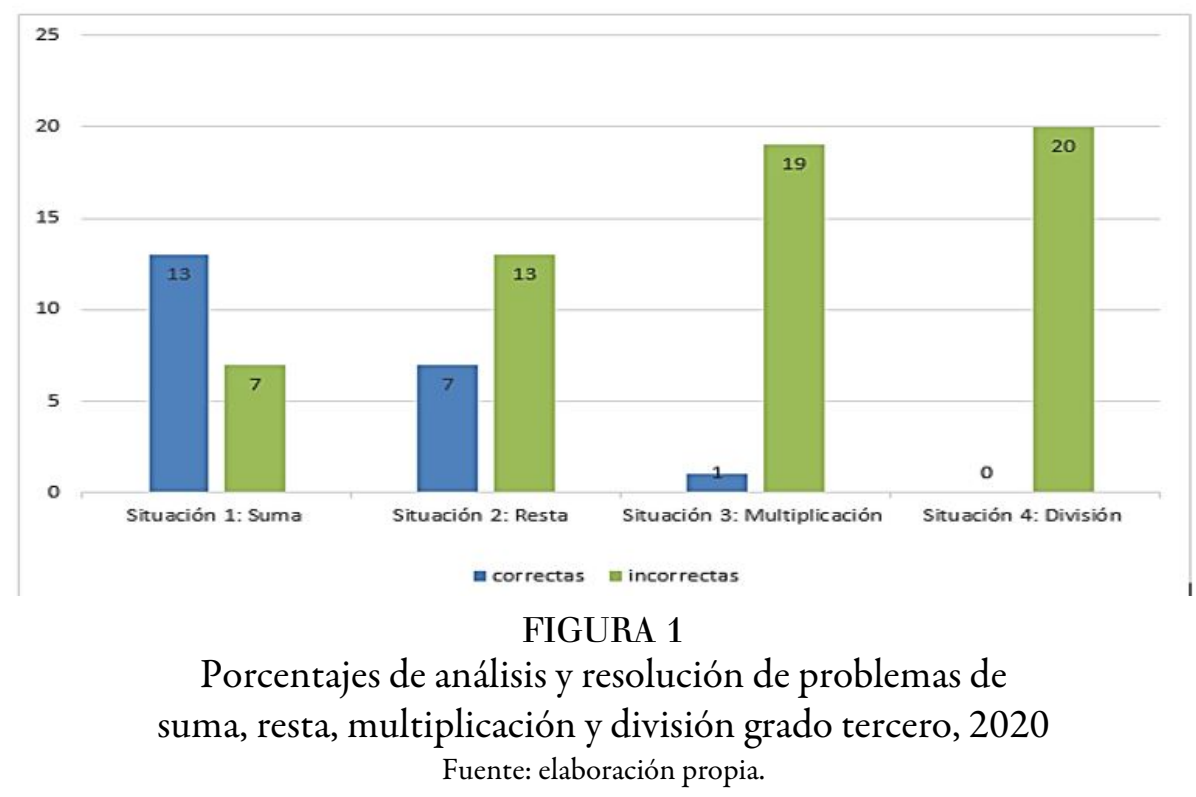

Con base en la Figura 1, se logró evidenciar que en la situación 1, el $65 \%$, correspondiente a 130 estudiantes, desarrollaron correctamente el problema; el $35 \%$, correspondiente a 70 estudiantes, lo desarrollaron mal. Dicha situación permite analizar que más de la mitad de estudiantes del grado tercero se les facilita desarrollar problemas que integran la suma, pero, aun así, hay estudiantes que presentan dificultades en este proceso, pues no logran identificar, con base en la lectura de la situación dada, la operación a desarrollar. Por lo tanto, es necesario implementar estrategias pedagógicas acordes a la edad para contribuir con el fortalecimiento.

En lo que hace referencia a la situación 2, se percibe que el $35 \%$, correspondiente a 70 estudiantes de grado tercero, analizó y resolvió de manera correcta la situación problema de resta; por el contrario, el $65 \%$, correspondiente a 130 estudiantes, presentó dificultad en la solución del este, lo que evidenció la complejidad en la manera en la que realizan el proceso de comprensión al momento de leer el problema y desarrollarlo.

Asimismo, como se muestra en la situación 3, se planteó un problema de multiplicación, cuyos resultados evidenciaron que el $5 \%$, es decir que 10 estudiantes, acertó en el desarrollo adecuado; el 95 $\%$, correspondiente a 190 estudiantes, no resolvió correctamente el problema debido a que presentan gran confusión en la terminología que permite identificar la operación y la forma pertinente para analizarlo, comprenderlo y resolverlo. Cabe aclarar que hay casos de estudiantes que, de acuerdo con su ritmo de trabajo dentro de las clases, evidencian poca agilidad y falta de atención, lo cual incide en dicha problemática.

Adicionalmente, en lo que evidencia la situación 4, es alarmante percibir que el $100 \%$ de estudiantes presentaron dificultad en el análisis y resolución del problema que involucra la división para ser desarrollado. Esto demuestra que existen diferentes factores que intervienen directamente en el proceso de enseñanza aprendizaje del estudiantado y en el rendimiento académico evidenciado en las acciones pedagógicas de las clases.

Es aquí donde surgió la inquietud sobre cómo fortalecer dichas competencias matemáticas mediante recursos TIC y "pensamiento computacional que permite y mejora la capacidad de resolver problemas, diseñar sistemas y comprender el comportamiento humano” (Wing, 2006, p.13), basados en el modelo 
STEAM para "propiciar el desarrollo de la competencia digital y el conocimiento del diseño de ingeniería” (Chien y Chu, 2017, p.5). También, se diseñó e implementó una estrategia basada en la programación para niños y niñas por medio de Scratch, incorporado en el AVA Moodle, en donde se aplicaron diferentes técnicas para la recolección y análisis de la información, con lo que se percibió el nivel de efectividad de la estrategia sobre el fortalecimiento de las competencias matemáticas en análisis y resolución de problemas.

Luego, en el diagnóstico inicial, basado en la aplicación de la encuesta de caracterización y una vez analizada y tabulada la información socio tecnológica, se logran determinar las necesidades pedagógicas y requerimientos para el diseño de la propuesta en el presente estudio. Se tomó en cuenta la implementación de herramientas dinámicas e interactivas para fortalecer la el desarrollo de las competencias propias de cada área del conocimiento por parte del estudiantado; por lo tanto, se evidenció la necesidad de utilizar unidades didácticas de aprendizaje con Scratch y Moodle para integrar herramientas innovadoras a la metodología de aprendizaje, y que estas permitieran clases más personalizadas acordes a las necesidades e intereses del alumnado, el fortalecimiento los conocimientos previos de las competencias matemáticas en análisis y la resolución de problemas que integran las cuatro operaciones básicas, esto para tener homogeneidad en el grupo y poder aplicar la herramienta propuesta.

El análisis realizado a la encuesta de caracterización socio tecnológica evidencia de forma clara las condiciones en las que se encuentra el estudiantado, la disponibilidad de recursos, el acompañamiento y la orientación recibida por parte del profesorado en el proceso de enseñanza aprendizaje.

En lo que se refiere a las condiciones y disponibilidad de recursos, se observa que hay algunos aspectos para tener en cuenta al momento de realizar el proceso de implementación, pues, aunque a cada estudiante se le dio en calidad de préstamo un computador portátil, una parte no cuenta con la conectividad suficiente y, de igual forma, no todas las personas acudientes pueden estar realizando el acompañamiento correspondiente por las distintas ocupaciones laborales. Al respecto, es necesario atender la flexibilización en los tiempos establecidos para el desarrollo de las actividades propuestas, de manera que no afecten el desarrollo académico y emocional del estudiantado.

De igual forma, en lo que tiene que ver con las orientaciones y explicaciones dadas por el profesorado en cada una de las clases, no se debe limitar a algunas temáticas específicas, sino, por el contrario, se evidencia en las respuestas que el grupo estudiantes se encuentra inmerso en el mundo de la tecnología; esto es fundamental para aprovechar el proceso de implementación de la propuesta y dar continuidad a esta en otros ambientes y espacios de aprendizaje que involucren el uso de los recursos con los que cuenta la institución y, si es necesario, se soliciten otros que faciliten la orientación de contenidos a partir de la interacción entre estos y el estudiantado.

Asimismo, al realizar el análisis de los resultados de la prueba diagnóstica y la prueba final, se logra evidenciar que el estudiantado del grado tercero de la IED Santa María logró fortalecer el desarrollo de las habilidades de análisis y comprensión de situaciones cotidianas de aprendizaje por medio de la integración curricular STEAM, que involucra las operaciones básicas, en coherencia con las competencias matemáticas y el pensamiento computacional. De igual forma, sobre las dificultades que presentaron al resolver las preguntas de la prueba diagnóstica en el razonamiento matemático, numérico, lógico y secuencial, se perciben grandes avances en la comprensión de los pasos y procedimientos correspondientes; así como la mejora de resultados, ya que, de acuerdo con la Figura 2, se evidencia mayor progreso en la discriminación de los resultados, lo cual permite tener una visión más significativa del progreso del estudiantado, eso quiere decir que los ejercicios y actividades implementados con la estrategia pedagógica Scratch, en el ambiente virtual de aprendizaje Moodle y realizados durante las unidades de aprendizaje, han permitido el progreso académico. 


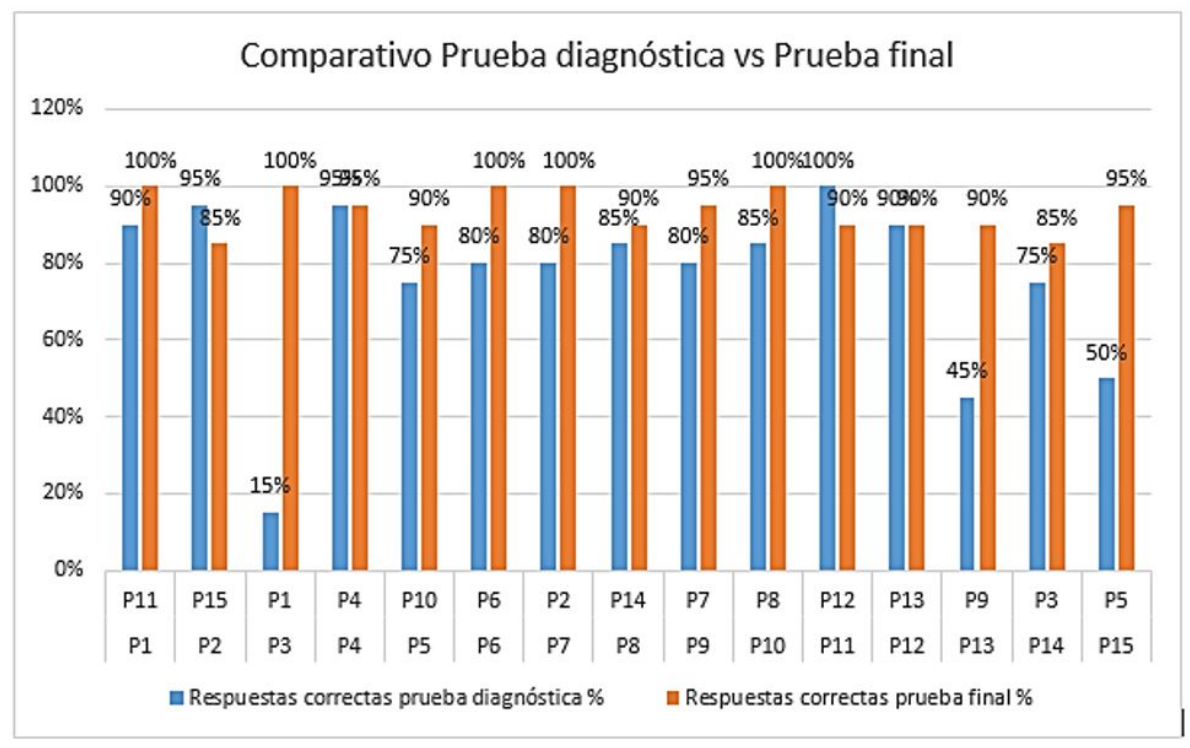

FIGURA 2.

Consolidado del comparativo de la prueba diagnóstica versus prueba final Fuente: elaboración propia.

\section{Consolidado del comparativo de la prueba diagnóstica versus prueba final}

De acuerdo con Figura 2 sobre el análisis comparativo del consolidado de la prueba diagnóstica con la prueba final, se logra evidenciar que el estudiantado mejoró el nivel de desarrollo de problemas que involucran las habilidades de pensamiento computacional, un aspecto favorable para la investigación, pues los resultados obtenidos en la aplicación de las dos pruebas no fueron bajos; por el contrario, se evidencia la calidad educativa institucional, en consideración del proceso de enseñanza aprendizaje que se lleva a cabo por medio de la mejora constante de las dificultades que se van presentando en el camino educativo. Es aquí donde la propuesta investigativa contribuye de manera significativa en el mejoramiento y fortalecimiento de dichos resultados, no solo en el área de matemáticas, tecnología e informática, sino también en las demás áreas del conocimiento.

Es necesario tener en cuenta que - al momento de aplicar la prueba diagnóstica de competencias matemáticas en análisis y resolución de problemas que integran las cuatro operaciones básicas y el pensamiento computacional por medio de la integración curricular STEAM al estudiantado de grado tercero-, al observar los resultados, se evidenciaron dificultades en análisis y comprensión lectora, habilidades matemáticas en operaciones básicas y razonamiento lógico. Además, se logra evidenciar un porcentaje considerable de respuestas incorrectas.

$\mathrm{Al}$ respecto, se debe tener en cuenta que, para el fortalecimiento de las competencias matemáticas y el pensamiento computacional, se diseñaron estratégicamente las unidades de aprendizaje con el fin de fundamentar la organización adecuada del desarrollo e implementación de la investigación, esto se retoma en cada una de las clases virtuales consecutivamente, para así poder afianzar procesos de aprendizaje. Por otra parte, se realizan actividades adicionales de forma conectada y desconectada para generar motivación frente a la competencia matemática, con la finalidad de observar y realizar un análisis de un antes y un después.

Por ello, con la implementación de la evaluación final, se logra evidenciar el progreso de cada estudiante en su forma de pensar y desfragmentar un problema a partir de las actividades realizadas de forma algorítmica.

La implementación de la estrategia pedagógica Scratch es "un entorno de programación que permite a niños y jóvenes crear sus propias historias interactivas, juegos y simulaciones y, a continuación, compartir esas creaciones en una comunidad en línea con otros jóvenes programadores de todo el mundo" (Resnick et al., 2009); la plataforma Moodle, que permite "administrar los contenidos según la 
necesidad pedagógica” (Hernández, G., 2015, p. 5), dio la opción al estudiantado de no solo programar y crear situaciones animadas matemáticas con Scratch, sino también de resolver problemas. En este sentido, de acuerdo con López (2014), el alumnado activó estrategias cognitivas y además usan

recursos y conceptos del pensamiento computacional para poder resolverlos. Además, se determinó que Scratch contribuye no solo al uso y apropiación del pensamiento matemático algorítmico, sino que se erige como mediadora tanto para implementar la estrategia de solución de problemas basada en un proceso detallado, como para posibilitar que el estudiantado tenga la oportunidad de utilizar conceptos del pensamiento computacional. (p. 67).

Por tal motivo se fortalecieron las competencias matemáticas en análisis y solución de problemas mediante el diseño y desarrollo de la estrategia pedagógica $S c r a t c h$, el ambiente virtual de aprendizaje Moodle y el pensamiento computacional (algoritmos y procedimientos) con el modelo STEAM. Cabe resaltar que las herramientas utilizadas, en coherencia con Rúa y Atuesta (2015), "para la creación del ambiente virtual de aprendizaje fueron de licencia libre, lo cual hizo posible la creación de estos ambientes sin la necesidad de adquirir software o la compra de un hospedaje web para su instalación” (p.33); esto contribuyó de manera significativa con el proceso de enseñanza y aprendizaje.

Asimismo, fue coherente con los planteamientos de la hipótesis inicial, la cual planteaba que con la estrategia propuesta se fortalecen las competencias en análisis y resolución de problemas que integran las operaciones matemáticas básicas mediante implementación de las TIC Scratch, Moodle y el pensamiento computacional en el estudiantado de tercero de primaria. Al realizar todo el proceso de implementación, desde el comienzo hasta el final del desarrollo de las cinco actividades incluidas en las dos unidades de aprendizaje diseñadas con los temas específicos, entre el $70 \%$ y el $90 \%$ de las 200 estudiantes, como se evidencia en la Figura 3, logran un alto desempeño en el desarrollo de las competencias matemáticas al resolver situaciones aditivas, multiplicativas y de secuencias numéricas lógicas que contribuyen con el fortalecimiento de las dificultades en este aspecto; esto según las matrices de referencia, estándares básicos de competencias y derechos básicos de aprendizaje que orientan los procesos y logros específicos que debía alcanzar el estudiantado en dicha competencia.

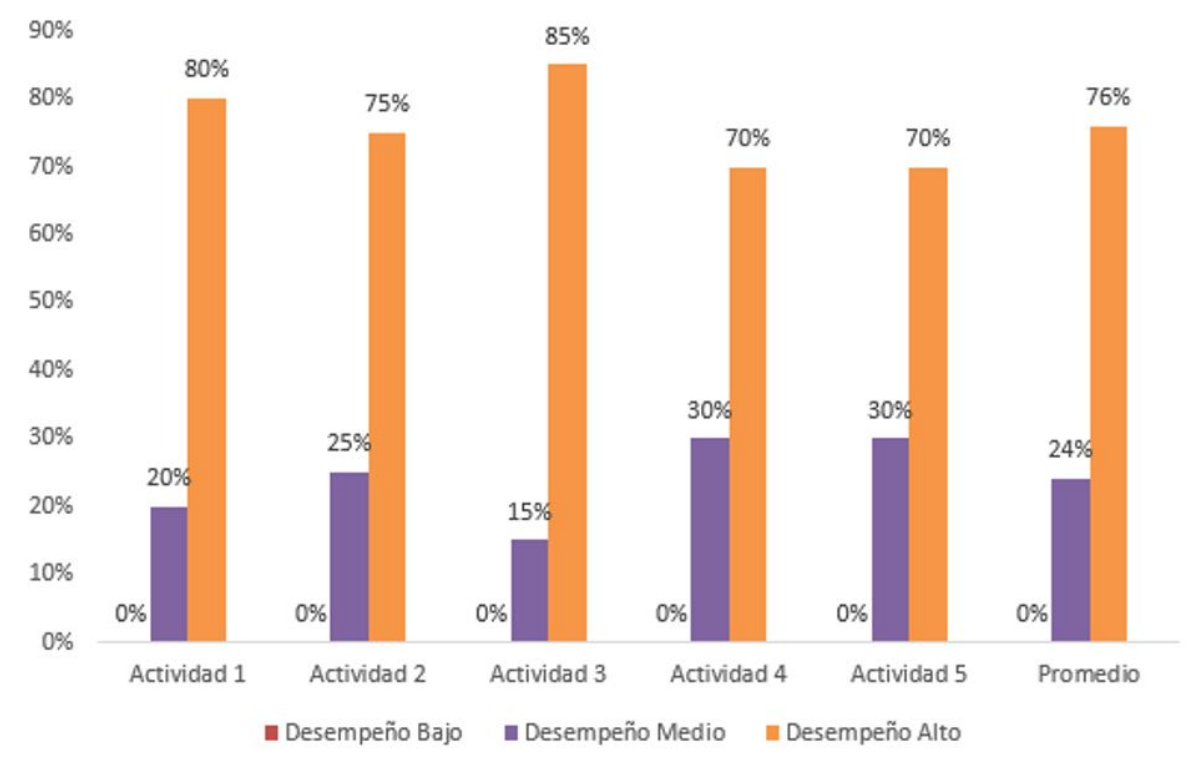

FIGURA 3.

Consolidado de resultado de las actividades Fuente: Elaboración propia.

En la Figura 3 se observa el consolidado final de proceso de evaluación de las cinco actividades, en coherencia con los criterios establecidos en las rúbricas y relacionados con el proceso de análisis y 
comprensión de problemas que involucran las cuatro operaciones básicas y el desarrollo del pensamiento computacional por medio del modelo STEAM y el desempeño individual (bajo, medio y alto) de cada estudiante. Al respecto, en lo que corresponde al desempeño bajo (0-2.9), ninguna de las 200 personas estudiantes forma parte de este nivel. También, se evidenció que entre el $15 \%$ y $30 \%$ presentan un desempeño medio (3.0-4.0), el cual tiene que ver con la dificultad mínima que presentan al desarrollar situaciones problema de forma desconectada y conectada, relacionados con el razonamiento numérico, lógico y secuencial matemático, y por medio del pensamiento computacional, en lo que hace referencia a los algoritmos y procedimientos específicos de cada problema de suma, resta, multiplicación y división.

Cabe resaltar que, inicialmente, la estrategia se diseñó para ser desarrollada en el aula de clase, pero dada la situación de la actual emergencia sanitaria Covid-19 se implementó en cada uno de los hogares del estudiantado, para el fortalecimiento de las competencias matemáticas y pensamiento computacional en análisis y resolución de problemas que integran las cuatro operaciones básicas. $\mathrm{Al}$ respecto el $90 \%$ de estudiantes, en los resultados arrojados en el instrumento de evaluación o encuesta de satisfacción, consideró interesante y pertinente el desarrollo de la propuesta investigativa para generar continuidad en el proceso de enseñanza y aprendizaje.

Es necesario tener en cuenta que también se determinó la efectividad de la estrategia implementada -mediante la valoración de actividades y evaluación de las competencias matemáticas y pensamiento computacional del alumnado- en el análisis y resolución de problemas que involucran las operaciones básicas, con la estimación de un porcentaje correspondiente al $85 \%$ que afirma la posibilidad de que se dé continuidad a la propuesta, ya que fue efectiva y motivante para aprender y fortalecer las competencias matemáticas y el pensamiento computacional.

De acuerdo con Guzmán y López (2009), se afirma que:

Se cumplió con las expectativas proyectadas, pues más del $90 \%$ del estudiantado presentaron un alto compromiso y se mostraron receptivas a los nuevos conceptos propuestos y desarrollados durante el cronograma socializado. Se evidenció un cambio de actitud hacia los algoritmos y el concepto de la programación y el pensamiento computacional por parte de las estudiantes, pasando de pensar en que era algo supremamente difícil a asimilar los conceptos de una manera sencilla, cambiando ese paradigma. Ahora piensan que es más fácil realizar un algoritmo empleando su propia lógica, además muestran un entusiasmo elevado en términos generales del grupo seleccionado. (p. 19-20)

Por otro lado, los datos recolectados permitieron afirmar que los objetivos propuestos se han logrado con suficiencia, pues se comprobó que da un $95 \%$ de efecto positivo, lo generó en el estudiantado bastante motivación y entusiasmo. En lo que tiene que ver con la utilización de la herramienta Scratch, el 85 \% de estudiantes lograron utilizarla de forma rápida, para la creación de las actividades sugeridas y necesitó pocas explicaciones al respecto. Esto confirmó que el estudiantado, sin tener experiencia en la creación de actividades por medio de la programación, pudo resolver situaciones cotidianas de aprendizaje en coherencia con las competencias matemáticas y el pensamiento computacional.

De igual modo, el interés y el entusiasmo mostrado por las clases virtuales fue de un aspecto que llena las expectativas generadas, esto se demostró en los resultados del instrumento de evaluación del alumnado, en el que hace una apreciación de gusto por las actividades. El 80 \% comenta que le ha llamado la atención el aprendizaje de los algoritmos y procedimientos para resolver problemas matemáticos que involucran las cuatro operaciones básicas y para crear situaciones y animaciones de aprendizaje con Scratch en la plataforma Moodle. Además del gusto e interés mostrados, refirió que es útil y divertida, porque en los encuentros sincrónicos el grupo de estudiantes expresó que les gustó la clase porque se trabajó de manera articulada las matemáticas con aplicaciones y herramientas tecnológicas, lo que resultó un tema novedoso e interesante.

En este sentido, Guzmán y López (2019) afirman que fue importante:

asumir nuestro rol de docentes activos en los procesos de enseñanza-aprendizaje generando ambientes propicios de adquisición de saberes y conocimientos con lo que haya a nuestro alrededor, ya que se 
promovieron este tipo de habilidades en el estudiantado, especialmente formar para pensar de una manera algorítmica y numérica secuencial, representadas por un conjunto de instrucciones organizadas que puedan dar solución a una tarea correctamente. (p.175)

De igual forma, cabe resaltar, desde la perspectiva de Guzmán y López (2019), que el pensamiento computacional implica:

un proceso cognoscitivo aplicado a la resolución de problemas de acuerdo a un contexto y haciendo uso de los conceptos fundamentales de la informática, fue importante fomentar y partir de este pensamiento ejercitar las habilidades en las estudiantes, las cuales son: creatividad e innovación, pensamiento crítico y colaboración, que conllevan al logro de resultados satisfactorios de aprendizaje, ya que se pudieron desarrollar diferentes habilidades como la lógica matemática y la comprensión lectora a través del aprendizaje del seguimiento de los algoritmos y la programación. Para ello se debe realizar una planeación que implica actividades de programación con ejercicios que sean intuitivos y llamativos para las estudiantes. (p. 175)

Por tal motivo, y como se realizó en la actividad 1 sobre la práctica inicial desconectada, Guzmán y López (2019) afirman que:

los algoritmos y procedimientos son usados en todos los aspectos de la vida, están presentes en todo elemento y en todo lugar, partiendo desde las actividades cotidianas hasta en artículos tecnológicos. Aunque es una palabra desconocida para la mayoría, se basa en el proceso para desarrollar problemas matemáticos inicialmente, pues es más sencillo de comprender para un estudiante de primaria, empleando ejemplos sencillos para seguir los pasos y realizar distintas actividades (recetas de cocina, oficios y actividades diarias, entre otros). (p. 84)

Finalmente, la ventaja de fortalecer las competencias matemáticas y el pensamiento computacional — por medio del lenguaje de programación para el desarrollo de habilidades matemáticas numéricas en las estudiantes de grado tercero de primaria de la Institución Educativa Departamental Santa Marí a- es que permitió que el estudiantado participante fuera consciente de su conocimiento, "generando aprendizaje que permite conocerse a sí mismas y conocer el entorno, enriqueciendo su proceso de pensamiento, siendo capaz de dar soluciones a problemas inmediatos de su propio entorno" Guzmán y López (2019) (p.178-179). Por tal motivo, estos resultados tan satisfactorios impulsan a continuar con la propuesta, de manera que se enriquezca, extienda y difunda para que se convierta en una estrategia integrada a las clases.

\section{Conclusiones}

A partir de los resultados y datos obtenidos y analizados, pudo concluirse que, de manera global, el curso Pensamiento computacional y resolución de problemas matemáticos con Scratch en Moodle, de acuerdo con el instrumento de evaluación aplicado, fue una experiencia significativa, que desarrolló con éxito cada uno de los objetivos propuestos específicamente en cada unidad de aprendizaje, esto con base en el consolidado del comparativo de las prueba diagnóstica y final.

De igual forma, es necesario tener en cuenta que el estudiantado presentaba falencias al momento de analizar y asociar las operaciones matemáticas con los problemas de la vida cotidiana, por lo que se hizo necesaria la implementación de cada uno de los componentes de la propuesta investigativa para facilitar la apropiación de ejercicios interactivos y desarrollo de actividades lúdicas, donde se manipula material y recursos concretos para la apropiación de conceptos y aplicación de las cuatro operaciones matemáticas básicas a través de situaciones de integración curricular STEAM, lo cual ayuda al alumnado a tomar las decisiones pertinentes para la resolución de cada problema.

Así mismo, se tuvo en cuenta que para el fortalecimiento de las competencias matemáticas y el pensamiento computacional se diseñaron estratégicamente las dos unidades de aprendizaje. La finalidad fue fundamentar la organización adecuada del desarrollo e implementación de la investigación, las cuales se retoman en cada una de las clases virtuales consecutivamente, para así poder afianzar procesos de aprendizaje. 
También, se realizan actividades adicionales de forma conectada y desconectada para generar motivación frente a la competencia matemática, para observar y realizar un análisis de un antes y un después. Es así que, con la implementación de la evaluación final, se evidenció el progreso de cada estudiante en su forma de pensar y desfragmentar un problema a partir de las actividades realizadas de forma algorítmica.

En consecuencia, se obtuvo un ambiente de aprendizaje virtual soportado por componentes tecnológicos referidos a la estrategia pedagógica con Scratch, en la plataforma Moodle, donde el grupo de estudiantes interactuó con situaciones de aprendizaje basado en la teoría constructivista de Piaget y la "construcción de la inteligencia abstracta" (Waldegg, 2002, p.33), con el propósito de que comprendieran elementos asociados a la competencia matemática y computacional a partir de situaciones que se encuentran en contexto.

Además, la investigación se constituyó como un ejemplo de aplicación de la metodología STEAM, ya que permitió la creación de un ambiente virtual de aprendizaje para fortalecer las prácticas pedagógicas, no solo de forma presencial, sino también en la modalidad virtual, lo cual facilitó la implementación y creación de otros ambientes de aprendizaje con características similares, relacionados con el Aprendizaje Basado en Problemas y en consideración de que es una estrategia "centrada en el estudiante, en la que este aprende a través de la experiencia de resolución de problemas" (Triantafyllou y TimcenK, 2013, pp. 1-8). Cabe resaltar que, en coherencia con los planteamientos de la hipótesis inicial, se fortalecen las competencias en análisis y resolución de problemas que integran las operaciones matemáticas básicas, esto mediante implementación de las TIC como Scratch y Moodle, y el pensamiento computacional.

También se determinó la efectividad de la estrategia implementada mediante la valoración de actividades y evaluación de las competencias matemáticas y pensamiento computacional del estudiantado, esto en cuanto al análisis y resolución de problemas que involucran las operaciones básicas, con la estimación de un porcentaje correspondiente al $85 \%$ de estudiantes que afirma la posibilidad de que se dé continuidad a la propuesta, ya que fue efectiva y motivante para aprender y fortalecer las competencias matemáticas y el pensamiento computacional.

En coherencia con la perspectiva de Guzmán y López (2019), cabe resaltar que:

de acuerdo con las temáticas propuestas desde el inicio se cumplió con las expectativas proyectadas, pues más del $90 \%$ de las estudiantes presentaron un alto compromiso y se mostraron receptivas a los nuevos conceptos propuestos y desarrollados durante el cronograma socializado. Se evidencia un cambio de actitud hacia los algoritmos y el concepto de la programación y el pensamiento computacional por parte de las estudiantes, pasando de pensar en que era algo supremamente difícil a asimilar los conceptos de una manera sencilla, cambiando ese paradigma. Ahora piensan que es más fácil realizar un algoritmo empleando su propia lógica, además muestran un entusiasmo elevado en términos generales del grupo seleccionado. (p.174).

Por otro lado, los datos recolectados permiten afirmar que los objetivos propuestos han sido logrados con suficiencia, pues se pudo comprobar un $95 \%$ de efecto positivo, ya que generó en el estudiantado bastante motivación y entusiasmo. En lo que tiene que ver con la utilización de la herramienta Scratch, el $85 \%$ de estudiantes logró utilizarla de forma rápida para la creación de las actividades sugeridas y necesitó pocas explicaciones al respecto. Esto confirma que el alumnado, sin tener experiencia en la creación de actividades por medio de la programación, pudo resolver situaciones cotidianas de aprendizaje en coherencia con las competencias matemáticas y el pensamiento computacional.

De igual modo, "el interés y el entusiasmo mostrado por las clases virtuales fue de un aspecto que llenó las expectativas generadas, esto se demuestra en los resultados del instrumento de evaluación al estudiantado, ya que evidencian una apreciación de gusto por las actividades" Guzmán y López (2019). El 80 $\%$ comentan que les ha llamado la atención el aprendizaje de los algoritmos y procedimientos para resolver problemas matemáticos que involucran las cuatro operaciones básicas y para crear situaciones y animaciones de aprendizaje con Scratch en la plataforma Moodle.

De acuerdo con Guzmán y López (2019) es necesario tener en cuenta que: 
además del gusto e interés mostrados, refieren que es útil y divertida, porque en los encuentros sincrónicos, expresan que les gusta la clase debido a que se trabaja de manera articulada las matemáticas con aplicaciones y herramientas tecnológicas, siendo este un tema novedoso e interesante. (p.21)

También, según Guzmán y López (2019), se puede afirmar que:

Es importante "asumir nuestro rol de docentes activos en los procesos de enseñanza-aprendizaje generando ambientes propicios de adquisición de saberes y conocimientos con lo que haya a nuestro alrededor" ya que es importante promover este tipo de habilidades en las estudiantes, especialmente formar para pensar de una manera algorítmica y numérica secuencial, representadas por un conjunto de instrucciones organizadas que puedan dar solución a una tarea correctamente. Teniendo en cuenta que el pensamiento computacional implica un proceso cognoscitivo aplicado a la resolución de problemas de acuerdo a un contexto y haciendo uso de los conceptos fundamentales de la informática, es importante fomentar y partir de este pensamiento ejercitar las habilidades en las estudiantes, las cuales son: creatividad e innovación, pensamiento crítico y colaboración, que conllevan al logro de resultados satisfactorios de aprendizaje, ya que se pueden desarrollar diferentes habilidades como la lógica matemática y la comprensión lectora a través del aprendizaje del seguimiento de los algoritmos y la programación. Para ello se debe realizar una planeación que implica actividades de programación con ejercicios que sean intuitivos y llamativos para las estudiantes. (p.175)

Por consiguiente, el desarrollo de la presente investigación permitió el fortalecimiento de las competencias matemáticas, específicamente en el desarrollo de problemas que involucraron las operaciones matemáticas básicas mediante implementación de las TIC Scratch, Moodle y el desarrollo del pensamiento computacional. Lo anterior a partir de la innovación y aplicación de acciones pedagógicas significativas y basadas en la articulación de conocimientos propios del nivel educativo de la población intervenida.

\section{Agradecimientos:}

Este trabajo de investigación, se ha realizado gracias a Dios, al Ser Supremo que brindó la posibilidad de llevar a cabo esta maestría y me orientó con sabiduría en la elaboración y ejecución del presente trabajo de grado.

Al tutor Sergio Albeiro Ramírez Gamboa, por ayudarme a percibir las cosas con calidad y exigencia, por darme esa seguridad en mi misma y mi trabajo, que sin ella esto no hubiese sido posible.

A la Institución Educativa Departamental Santa María, padres y madres de familia, y el estudiantado, quienes fueron parte esencial de este trabajo y actantes principales del proceso.

A mi familia por todo su apoyo, a mis compañeras de grado tercero y a todas las personas que de diferentes formas colaboraron e hicieron posible la realización de este trabajo de investigación.

\section{REFERENCIAS BIBLIOGRÁFICAS}

Acuña-Medina, N., León-Arias, M., López-Palomino, L., Villar-Navarro, C. y Mulford-León, R. (2018). Aprendizajes de las Matemáticas Mediados Por Juegos Interactivos En Scratch En La IEDGVCS. Cultura Educación $Y$ Sociedad, 9(2), 32 - 42. https://doi.org/10.17981/cultedusoc.9.2.2018.03

Álvarez, M. (2017). Desarrollo del pensamiento computacional en educación primaria: una experiencia educativa con Scratch. Castilla. Revista de Ciencias de la Educación. (2), 45-64. https://revistes.urv.cat/index.php/ute/article /view/1820

Arias, F. (2012). El proyecto de investigación. Introducción a la metodología cientifica. Episteme

Aristizabal, J.H. (2016). El juego como una estrategia didáctica para desarrollar el pensamiento numérico en las cuatro operaciones básicas. Colombia. Sophia 12(1), 117-125.

Basogain-Olabe, X., Olabe-Basogain, M. y Olabe-Basogain, J. (2015). Pensamiento Computacional a través de la Programación: Paradigma de Aprendizaje. Revista de Educación a Distancia (RED), (46). https://revistas.um.e s/red/article/view/240011 
Mercy Liceth Cabra Páez, et al. Desarrollo del pensamiento computacional y las competencias matemá...

Barbarán, J. (2014). Resolver problemas para aprender matemáticas. Revista digital educativa. (2011), 31-35. http:// www.ugr.es/ barbaran/Descargas/Resolver\%20problemas\%20para\%20aprender\%20matematicas.pdf

Chien, Y.H. y Chu, P.Y. (2017). Construcción de un marco teórico para el enfoque STEAM en la Educación Primaria. Martinez Losada.

Creswell, J.W. (2009). Técnicas de Investigación Cualitativa y Mixta. Mc Graw Hill.

Espinosa. (2014). Evaluación de competencias mediante rúbrica. Importancia de las matemáticas en la evaluación de competencias genéricas. Europa: Complutense. Historia y Comunicación Social, (18), 243-255. https://doi.org/1 0.5209/rev_HICS.2013.v18.44240

González, C.S. (2019). Estado del arte en la enseñanza del pensamiento computacional y la programación en la etapa infantil. Universidad Salamanca.

Granados, L. (2019). El programa Scratch y la resolución de problemas en el área de matemática de los estudiantes de una institución educativa nivel primario. Perú. http://repositorio.unh.edu.pe/handle/UNH/2424

Guzman, E. y López, W. (2019) Implementación de una estrategia didáctica de programación para la formación de habilidades de resolución de problemas en niños. Tesis de Maestría inédita. Universidad Distrital Francisco José de Caldas.

Halmos, P. R. (1980). The Heart of Mathematics. American Mathematical Monthly, 87(7), 519-524. https:// doi.org/10.1080/00029890.1980.11995081

Hernández, G. (2015). Análisis del uso y manejo de la plataforma Moodle en docentes de matemáticas, para el desarrollo de competencias integrales en estudiantes de primaria. Revista Q, 10(19). 10. Colombia. https://bit.ly/3iU7zYB

Hernández-Sampieri, R. y Mendoza, M. (2008). Metodología de la Investigacion. McGraw Hill.

Lagos, S. (2017). Propuesta de intervención de aula para favorecer el desarrollo del pensamiento numérico, a partir de situaciones de estructura aditiva en estudiantes de ciclo I. Colombia. http://hdl.handle.net/10818/19991

López, J. (2014). Actividades de aula con Scratch que favorecen el uso del pensamiento algorítmico. Tesis de Maestría inédita. Universidad ICESI.

Mendoza, M. (2017). Software de programación "Scratch" en el desarrollo del pensamiento lógico matemático de estudiantes de una institución educativa primaria [tesis de maestría]. Universidad Cesar Vallejo

Ministerio de Educación Nacional [MEN]. (2016). Derechos Básicos de Aprendizaje. Mineducación.

Pérez, M. (2017). El pensamiento computacional para potenciar el desarrollo de habilidades relacionadas con la resolución creativa de problemas. 3C TIC. Cuadernos De Desarrollo Aplicados a Las TIC, 6(1), 38-63. https:/ /doi.org/10.17993/3ctic

Quintero, J. (2019). Influencia del Programa Scratch en el Pensamiento Computacional en Estudiantes del Nivel Primario, Tacna 2018. Perú. http://repositorio.upt.edu.pe/handle/UPT/1015

Resnick, M., Maloney, J., Monroy, A., Rusk, N., Eastmond, E., Brennan, K., Millner, A., Rosenbaum, E., Silver, J., Silverman, B. y Kafai, Y. (2009). Scratch: programación para todos. Communications of the ACM. Revista Ciencias de la Educación, 52(11), 60-67. http://cacm.acm.org/magazines/2009/11/48421-scratch-programmi ng-for-all/fulltext

Rios, G. (2015). Scratch + ABP, como estrategia para el desarrollo del pensamiento computacional [tesis de maestría inédita]. Universidad EAFIT.

Rúa, M. y Atuesta M. (2015). Diseño de un ambiente virtual de aprendizaje para el fortalecimiento de la formulación y solución de problemas asociados al pensamiento Variacional. Universidad EAFIT. http://hdl.handle.net/1078 $4 / 8194$

Suarez, G. (2019). Recursos educativos digitales en el desarrollo del pensamiento lógico matemático [tesis de maestría inédita]. Universidad de Guayaquil. http://repositorio.ug.edu.ec/handle/redug/40615

Triantafyllou, E. y TimcenK, O. (2013). Applying Constructionism and ProblemApplying Constructionism and Problem Based Learning for Developing Dynamic Educational Material for Mathematics at Undergraduate University Level. International Research Symposium on Problem-Based Learning (IRSPBL), (428), 1-8. https://bit.ly/3x YoMV4 
Vivas, C., Murillo, Z., y Christancho, J. (2019). Scratch. Estrategia didáctica para el aprendizaje de las tablas de multiplicar en escuela nueva. Educación Y Ciencia, (20), 43-60. https://revistas.uptc.edu.co/index.php/educac ion_y_ciencia/article/view/8897

Waldegg, G. (2002). El uso de las nuevas tecnologias para la enseñanza y el aprendizaje de las ciencias. Mexico: REDIE. Revista Electrónica de Investigación Educativa, 4(1). https://bit.ly/2Uq93R0

Wing, J. M. (2006). Computational Thinking. Communications of the ACM. Revista de Educación a Distancia. (49), 33-35 https://doi.org/10.1145/1118178.1118215

\section{INFORMACIÓN ADICIONAL}

Cómo citar: Cabra-Páez, M. L. y Ramírez-Gamboa, S. A. (2022). Desarrollo del pensamiento computacional y las competencias matemáticas en análisis y solución de problemas: una experiencia de aprendizaje con Scratch en la plataforma Moodle. Revista Educación, 46(1). http://doi.org/10.15517/revedu.v46i1.44970 